\title{
Exploring Information Delivery on a Guided Tour Using Mobile Projection and Visual Markers
}

\author{
Jonna Häkkilä ${ }^{1}$, Lasse Virtanen ${ }^{1}$, Juho Rantakari ${ }^{1}$, Ashley Colley ${ }^{1}$, Keith Cheverst ${ }^{2}$ \\ ${ }^{1}$ University of Lapland \\ Yliopistokatu 8 \\ 96300 Rovaniemi, Finland \\ firstname.lastname@ulapland.fi \\ ${ }^{2}$ Lancaster University \\ LA1 4WA UK \\ Lancaster, UK \\ k.cheverst@lancaster.ac.uk
}

\begin{abstract}
We present an in-the-wild user study $(n=27)$ investigating the combination of two mobile technologies picoprojectors and marker based information browsing. We studied a tour, where the tour guide used combinations of fixed and projected elements to present information, and compare four cases: A) as a baseline, a traditional paper poster, B) a projected poster, C) a printed paper fiducial marker, viewed through a mobile device browser application, and D) a projected fiducial marker viewed through a mobile device browser application. As a contribution, we present a novel approach to ad hoc projection of markers, and the findings of the user study. Here, the salient findings suggest that the techniques using markers have the potential to enhance tour participants' experience, but face practical challenges due to lighting conditions and image stability.
\end{abstract}

\section{Author Keywords}

Markers; mobile projection; mobile phones; tour guides; user studies.

\section{ACM Classification Keywords}

H.5.m. Information interfaces and presentation (e.g., $\mathrm{HCI}$ ): Miscellaneous.

\section{INTRODUCTION}

Many museums and exhibitions nowadays provide the possibility for visitors to discover additional information on exhibits using mobile phones or tablets. One common approach to this is the use of visible markers, e.g. QR codes, which are viewed through the mobile device and open e.g. a web view that may include multimedia content. An alternative, traditional way for visitors to gain deeper knowledge about exhibits is through a guided tour with a human guide, providing explanatory commentary.

Permission to make digital or hard copies of all or part of this work for personal or classroom use is granted without fee provided that copies are not made or distributed for profit or commercial advantage and that copies bear this notice and the full citation on the first page. Copyrights for components of this work owned by others than ACM must be honored. Abstracting with credit is permitted. To copy otherwise, or republish, to post on servers or to redistribute to lists, requires prior specific permission and/or a fee. Request permissions from Permissions@acm.org.

MUM'16, December 12-15, 2016, Rovaniemi, Finland

(C) 2016 ACM. ISBN 978-1-4503-4860-7/16/12\$15.00

DOI: http://dx.doi.org/10.1145/3012709.3012722

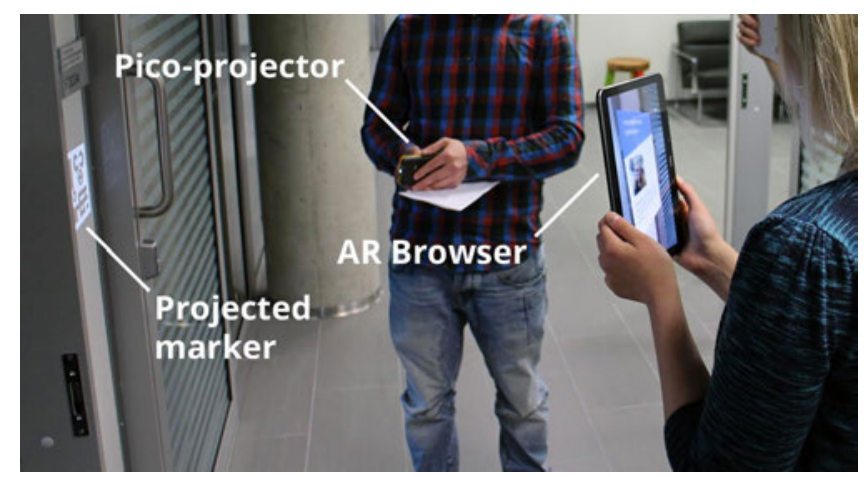

Figure 1. One of the information delivery methods evaluated. A tour guide uses a picoprojector to display a fiducial marker, which is then read by tour participants using a tablet device equipped with an AR browser application.

However, the human-guided tour does not easily integrate the ad hoc use of multimedia content. For example, the use of large screens would disturb other people in the area, or independent browsing on personal mobile devices could disturb the guided tour experience and divide attention between the tour guide and the additional content.

In this paper we present a study comparing four different methods of additional content delivery during a guided tour. As well as a traditional paper poster as a baseline, the solutions tested make use of handheld picoprojection, augmented reality (AR) browsing, and a combination of picoprojection and AR browsing (Figure 1). The aim of the study was to explore how the alternative techniques performed in the actual context of a guided tour. Here, we aimed to elicit information on how users perceived the projection- and AR based presentation techniques and how they affected the tour group dynamics and the relationship with the tour guide.

This work extends the initial results presented in [3], which introduced the projected marker approach, and presents the evaluation of its use, in-the-wild on a guided tour. Our user study provides information for practitioners creating such mobile AR tours e.g. in museum environments.

\section{RELATED WORK}

There is a large amount of existing research on handheld projectors in a variety of applications, and many potential areas of use have been suggested, see e.g. [8, 11, 16]. Rukzio et al. [11] have reported on the design space around 
personal projection, and Molyneaux et al. [8] report on studies using handheld projection both for infrastructurebased and infrastructure-less cases. Related to navigation, mobile projection has been used e.g. to augment maps [6] and to guide museum tours [13].

Visual markers such as QR codes provide visual affordance of the interaction possibilities. However, Mäkelä et al. [7] has pointed out that they give a somewhat technical impression and are not aesthetically pleasing. Augmented Reality (AR) marker based systems have been studied in the context of adding information to paper maps [3,12]. The AR based MapLens concept [9] examined augmenting a physical map to guiding groups, finding that the approach integrated the participants in the study group, improving cooperation in tasks. There have been a variety of studies on the use of QR codes in libraries and museums, e.g. [2] report on a QR code based children's game in a museum. Similarly, [5] report on the use of QR codes in outdoor learning environments. The low usage of $\mathrm{QR}$ codes deployed in a library environment is noted in [12]. Considering other types of physical tags, Hardy et al. report on an in-the-wild study on user customizable NFC tags [4], demonstrating the versatility of tag based approaches.

The combination of projection devices and fiducial markers to create a located information display is explored in [1], where a user could project exhibit related imagery onto the exhibit object itself. Our approach is somewhat opposite to this, in that we project the marker, which is then read by the tour audience using smartphones and tablets. This paper contributes by presenting, as far as we are aware, the first evaluation of the use of the projected marker technique on a guided tour. This differs from prior work that has e.g. reported on the use of static printed $A R$ markers in various education oriented contexts $[2,5,12]$.

\section{CONCEPT AND COMPARATIVE USER STUDY}

\section{Concept and Implementation}

In the projected markers concept, the tour guide uses a mobile phone integrated picoprojector to project a fiducial marker to a wall near an exhibit. Exhibition visitors then view the marker through their mobile phones to see multimedia content augmented on to the view. The augmented content is linked to the position and presence of the marker. The tour guide can dynamically select which marker to project, and where to project it, and hence control the content seen by the visitor group.

We implemented an AR viewer application using Unity 3D and the Vuforia AR plugin, deployed as an Android application. The fiducial markers were designed in Photoshop and optimised for recognition in low contrast conditions, i.e. when projected. As a handheld picoprojector we utilized the Samsung Galaxy Beam projector phone. The tour participants used a mixture of mobile devices to view the AR content (Samsung Galaxy Tab 4 tablets and Samsung Galaxy S5 and Motorola Moto G smartphones)

\section{Comparing Information Delivery Methods}

To provide data on the relative benefits of the projected marker solution vs. other approaches applicable to the same context, we designed a comparative user study. The study included 4 different methods of providing additional information to a guided tour audience, (A) a traditional paper poster, (B) a projected poster, (C) a printed AR marker, and (D) a projected AR marker (Figure 2). The actual content in all 4 formats was identical.
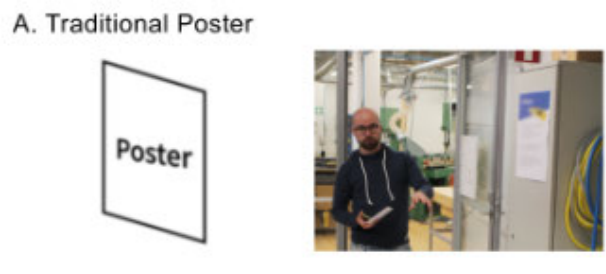

B. Projected Poster

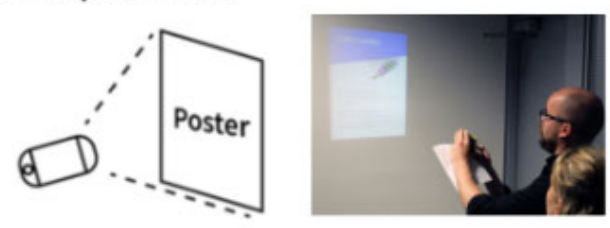

C. Paper Marker
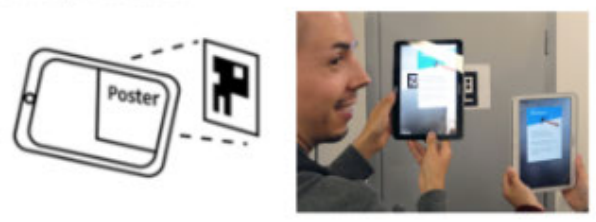

D. Projected Marker

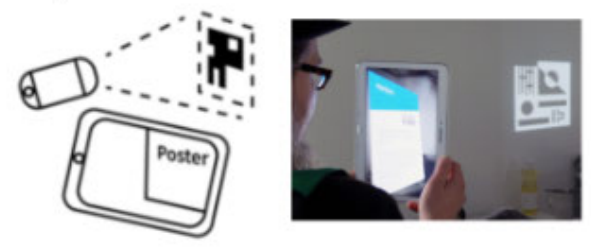

Figure. 2. Presentation techniques compared in the user study.

The study context consisted of guided tour at a university campus for new university students. Eight different pointsof-interest (POI) were situated around the university building and content for each was created. The content at each POI consisted of a few sentences of text and a picture. Each of the four presentation techniques A-D was used at 2 of the tour POIs.

\section{Study Procedure}

We recruited 27 test participants (14 male, 13 female). The participants' ages ranged from 18 to 37 years $(M=25, S D=$ 5.6). All the participants were students, owned a mobile phone and 26/27 owned a smartphone. Of the participants, 19 were familiar with augmented reality, but only 12 of them had used augmented reality applications or similar. Almost everyone (24/27) had used QR codes before, but only $5 / 27$ had used mobile projectors. The participants were divided into 6 tour groups with 3-6 participants per group. 
All the subjects experienced 2 of each of the 4 presentation techniques. The direction in which the groups completed the tour was counterbalanced to reduce learning effects.

The study procedure consisted of filling out background questionnaire, the guided tour itself (which lasted around 30 minutes), and completing a written survey. The participants rated each of the presentation methods against five different user experience criteria on a 7-point rating scale. Participants were also asked to select their preferred method, give free comments explaining their selection and to ideate different use cases for each presentation method. A researcher observed the tour, and took notes in situ. The location of the tour POIs is illustrated in Figure 3.

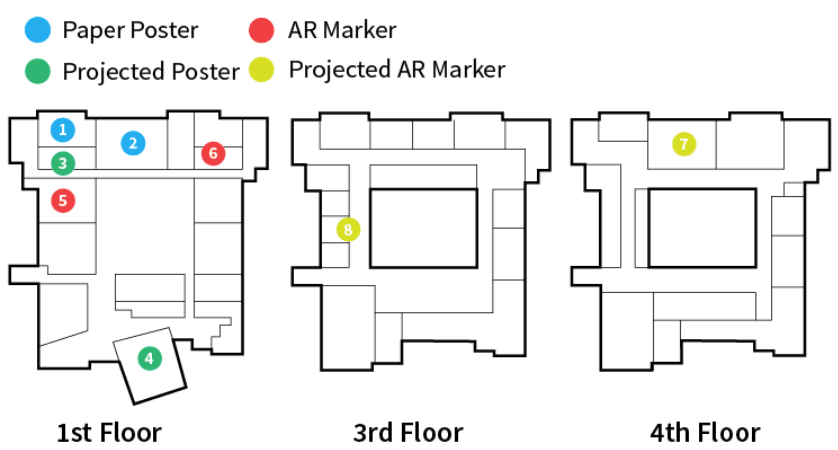

Figure 3. POI locations and types on the tour.

\section{RESULTS}

\section{Subjective Ratings}

Participants' ratings for the presentation techniques against the criteria; fun, easy, useful, interesting and versatile are shown in Figure 4. It can be noted that the novel presentation techniques were considered more fun and interesting, compared to the traditional paper poster. However, in terms of ease of use (easy), the paper poster was ranked highest. The paper marker solution (C) also scored somewhat higher in terms of its ease of use than the techniques involving projection (B \& D), with $74 \%$ of participants ranking it on the positive side. When asked to select their preferred presentation solution $41 \%$ selected the paper marker solution, $30 \%$ the paper poster, $19 \%$ the projected marker and $10 \%$ the projected poster.

\section{Qualitative Feedback}

The test participants' qualitative comments were analysed using an open coding approach. One researcher defined concept categories and a second researcher then mapped the comments to the defined categories (Table 1). The paper poster (A) received many positive comments regarding its availability i.e. that it did not require any special technology to view it. In this respect a participant commented: "This [paper poster] doesn't require a separate device to read the information and doesn't need any power. Posters are clear and easily accessible" (\#14).

On the other hand, participants commented that the paper poster presentation required a big physical space and that paper as a material was not seen as ecological compared to other presentation approaches. Clearly participants identified the paper format to be less adaptable than the other formats, receiving 5 negative comments in this respect vs. other formats which each received at least 9 positive comments relating to their adaptability. The attractiveness of paper posters was also commented negatively. Interestingly, one participant commenting that there are issues of 'display blindness' [11] also with paper posters, "Boring, and you might miss it if there's a lot of another information around." (\#27).

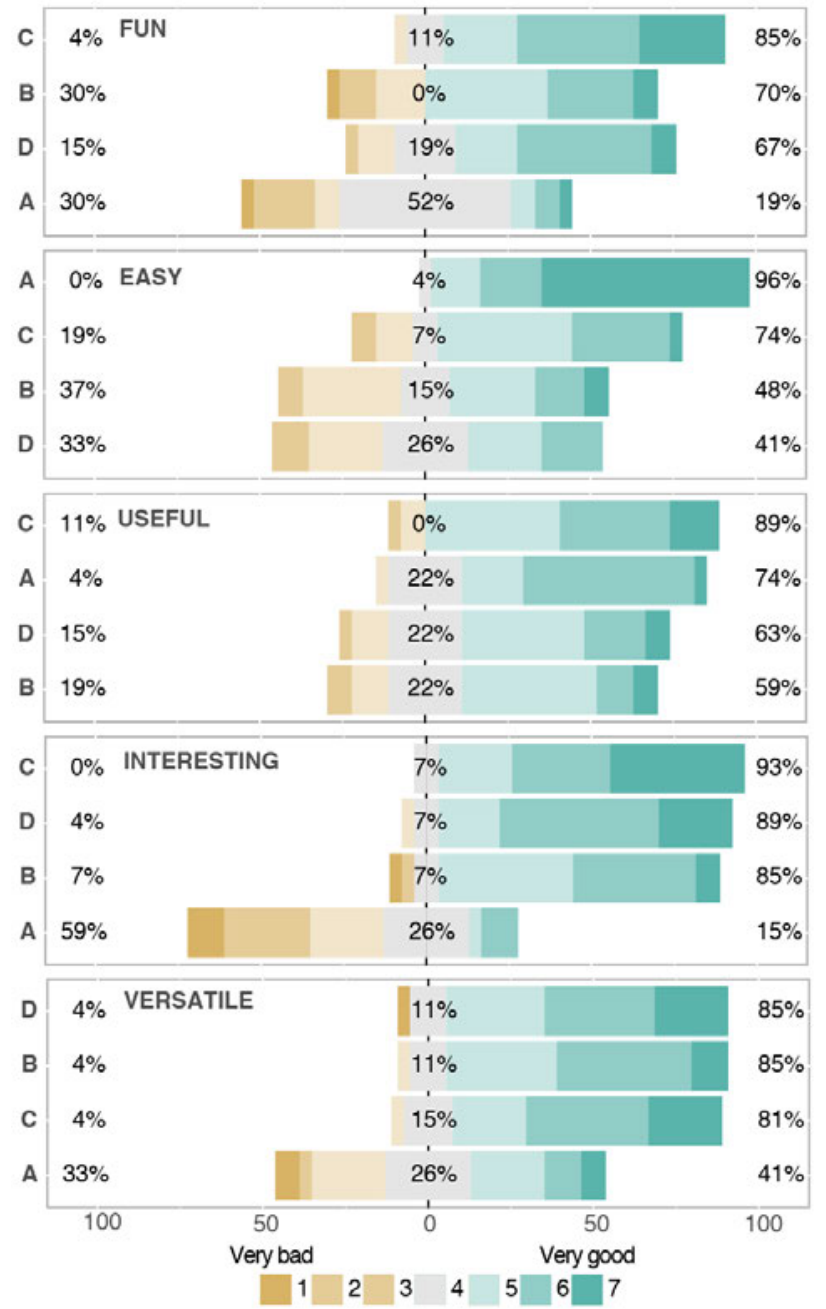

Figure 4. Ratings for each of the presentation formats on a 7point rating scale. $A=$ paper poster, $B=$ projected poster, $C=$ paper marker, $D=$ projected marker. Percentages refer to the number of responses on negative side, neutral and on positive side respectively.

The projected poster (B) was considered as adaptable, and received positive comments about its immateriality and mobility. One participant noting, "It's mobile, versatile and inspiring. It can be used in many situations and different places" (\#2). Also, it was seen as benefit that there was no need for the information to be in a fixed location, or for it to be visible all the time. Example comments being, "There's 
no need for paper and you can project poster to any flat surface" (\#7) and "No need to have all kinds of papers around, and you can see the information only when needed" (\#16). However, there were many negative comments about the perceived reliability of the projected poster solution. Issues here related to the reliability of the projector itself and visibility problems in a brightly lit environment, "If someone switches the light on in the room, it's hard to see information" (\#22).

\begin{tabular}{l|cccc}
\multicolumn{1}{c}{ Category } & $\begin{array}{c}\text { Paper } \\
\text { Poster } \\
\text { (A) }\end{array}$ & $\begin{array}{c}\text { Projected } \\
\text { Poster } \\
\text { (B) }\end{array}$ & $\begin{array}{c}\text { Paper AR } \\
\text { Marker } \\
\text { (C) }\end{array}$ & $\begin{array}{c}\text { Projected } \\
\text { AR Marker } \\
\text { (D) }\end{array}$ \\
\hline Attractiveness & $+2 /-8$ & $+5 /-4$ & $+10 /-6$ & $+11 /-5$ \\
Reliability & $+8 /-8$ & -19 & $+4 /-12$ & $+2 /-15$ \\
Availability & $+36 /-10$ & $+19 /-12$ & $+12 /-16$ & $+8 /-18$ \\
Adaptability & -5 & +9 & $+9 /-1$ & +12 \\
Novelty & -6 & $+1 /-1$ & +3 & \\
Uncategorized & & 2 & 2 &
\end{tabular}

Table 1. Open coding based analysis of participants' comments during and after the tour. Positive numbers $(+)$ indicate positive remarks, negative numbers represent negative remarks.

The paper marker based presentation format (C) was praised for its small need of physical space, accounting for positive comments in the categories of attractiveness and availability. Participants commenting e.g., "It takes only little space and is practical" (\#6). Participants also liked the possibility to update the content i.e. its adaptability Participant \#1 commenting, "The information requires just a little physical space and you can add other media as necessary". Similarly with the projector solution, negative comments were related to the requirement for electronic hardware, accounting for many negative comments in the categories of reliability and availability, "[It] requires a special electronic device to work and not all will have it" (\#14).

The projected marker solution (D) was also seen to suffer from its need for technology, some participants considering it complex, "It requires two devices. It's not very tempting" (\#26). It was considered positive that there was no need for any visible markings on the environment at all, increasing its attractiveness. In this respect participants commented, "This might work on tours and in bigger presentations as there is no need to attach information to the wall" (\#17), and "No pointless papers on the wall anymore" (\#3), "You can show information when needed. You can also control when that information is available and when isn't" (\#10). Additional problems highlighted with the reliability of the projected marker solution related to disruptions caused by users and environmental conditions for example shaky hands or a brightly lit environment: "The devices are shaking so the picture is shaking.” (\#6).

Focusing on the guided tour use case, many participants commented that the projected markers solution would be useful in guiding group situations. Specifically, museums or other historical locations were mentioned as applications. Here, it was noted by participants that the solution created the strongest link between the guide and audience and also supported cohesion between the tour audience.

\section{DISCUSSION}

From a purely functional viewpoint the traditional paper poster was clearly considered as the easiest solution, with highest availability, by the test participants. The remaining solutions were ranked approximately equally in terms of ease of use. However, considering more experiental aspects, the paper poster was seen as less fun and less interesting. In comparison both of the marker-based solution were rated strongly in terms of fun and interest, and they were commented to be attractive. The main limitation of the projector-based solutions was that the luminance of the handheld projector made it impossible to use the solutions in direct sunlight. The stability of the both the projector solutions was also challenging, and this was doubly so in the case of the projected marker case, where two parties contributed to the physical instability.

In terms of versatility the paper poster was considered limited, the projected poster and the two marker based solutions being considered as more versatile. Interestingly, some comments related to the paper poster concerned sustainability aspects, i.e. that it was using natural resources and would need to be thrown away and replaced if the content changed. The possibility to project markers in a variety of positions was seen as beneficial to allow flexibility to cope with different group sizes, and create a more intimate experience when the group contained fewer members.

We acknowledge that our work is limited by its single context and the limited number of test participants. However, as it was carried out in an authentic context, we believe our findings have validity for application to other environments and contexts.

\section{CONCLUSION}

We conducted an in-the-wild user study $(n=27)$ comparing methods to provide additional multimedia content to guided tour participants. Four methods were compared, a paper poster, a projected poster and printed and projected fiducial markers viewed through a mobile device AR browser. Overall, the paper fiducial marker based solution was preferred with $41 \%$ of participants selecting it as their preferred solution. The projected markers technique was considered to have potential to enhance the tour participants' experience, but would face challenges due to lighting conditions and image stability.

\section{ACKNOWLEDGEMENTS}

This research has been supported by a grant from Tekes the Finnish Funding Agency for Innovation as part of The Naked Approach - A World without Gadgets programme. 


\section{REFERENCES}

1. Liam Betsworth, Huw Bowen, Simon Robinson, and Matt Jones. 2014. Performative technologies for heritage site regeneration. Personal Ubiquitous Comput. 18, 7 (October 2014), 1631-1650. DOI: http://dx.doi.org/10.1007/s00779-014-0766-3

2. Ugo B. Ceipidor, Carlo M. Medaglia, Amedeo Perrone, Maria De Marsico, and Giorgia Di Romano. 2009. A museum mobile game for children using QR-codes. In Proceedings of the 8th International Conference on Interaction Design and Children (IDC '09). ACM, New York, NY, USA, 282-283. DOI=http://dx.doi.org/10.1145/1551788.1551857

3. Jens Grubert, Michel Pahud, Raphael Grasset, DieterSchmalstieg, and Hartmut Seichter. 2015. The utility of Magic Lens interfaces on handheld devices for touristic map navigation. Pervasive and Mobile Computing 18 (2015), 88 - 103.

4. Jonna Häkkilä, Juho Rantakari, Lasse Virtanen, Ashley Colley, and Keith Cheverst. 2016. Projected Fiducial Markers for Dynamic Content Display on Guided Tours. In Proceedings of the 2016 CHI Conference Extended Abstracts on Human Factors in Computing Systems (CHI EA '16). ACM http://dx.doi.org/10.1145/2851581.2892345

5. Robert Hardy, Enrico Rukzio, Paul Holleis, and Matthias Wagner. 2011. Mystate: sharing social and contextual information through touch interactions with tagged objects. In Proceedings of the 13th International Conference on Human Computer Interaction with Mobile Devices and Services (MobileHCI '11). ACM, New York, NY, USA, 475-484. DOI=http://dx.doi.org/10.1145/2037373.2037444

6. Lai, Hsin-Chih, Chun-Yen Chang, Li Wen-Shiane, YuLin Fan, and Ying-Tien Wu. 2013. The implementation of mobile learning in outdoor education: application of QR codes. British Journal of Educational Technology 44, no. 2 (2013): E57-E62. DOI: 10.1111/j.1467-8535.2012.01343.x

7. Markus Löchtefeld, Johannes Schöning, Michael Rohs, and Antonio Krüger. 2009. Marauders light: replacing the wand with a mobile camera projector unit. In Proceedings of the 8th International Conference on Mobile and Ubiquitous Multimedia (MUM '09). ACM, New York, NY, USA, Article 19, 4 pages. DOI=http://dx.doi.org/10.1145/1658550.1658569

8. Kaj Mäkelä, Sara Belt, Dan Greenblatt, and Jonna Häkkilä. 2007. Mobile interaction with visual and RFID tags: a field study on user perceptions. In Proceedings of the SIGCHI Conference on Human Factors in Computing Systems (CHI '07). ACM, New
York, NY, USA, 991-994.

DOI=http://dx.doi.org/10.1145/1240624.1240774

9. David Molyneaux, Shahram Izadi, David Kim, Otmar Hilliges, Steve Hodges, Xiang Cao, Alex Butler, and Hans Gellersen. 2012. Interactive environment-aware handheld projectors for pervasive computing spaces. In Proc. Pervasive'12, Springer, 197-215. DOI:10.1007/978-3-642-31205-2_13

10. Ann Morrison, Antti Oulasvirta, Peter Peltonen, Saija Lemmela, Giulio Jacucci, Gerhard Reitmayr, Jaana Näsänen, and Antti Juustila. 2009. Like bees around the hive: a comparative study of a mobile augmented reality map. In Proceedings of the SIGCHI Conference on Human Factors in Computing Systems (CHI '09). ACM, New York, NY, USA, 1889-1898. DOI=http://dx.doi.org/10.1145/1518701.1518991

11. Jörg Müller, Dennis Wilmsmann, Juliane Exeler, Markus Buzeck, Albrecht Schmidt, Tim Jay, and Antonio Krüger. 2009. Display blindness: The effect of expectations on attention towards digital signage. In International Conference on Pervasive Computing, pp. 1-8. Springer Berlin Heidelberg, 2009. DOI: 10.1007/978-3-642-01516-8_1

12. Michael Rohs, Johannes Schöning, Martin Raubal, Georg Essl, and Antonio Krüger. 2007. Map Navigation with Mobile Devices: Virtual versus Physical Movement with and without Visual Context. In Proceedings of the ninth international conference on Multimodal interfaces - ICMI '07. ACM Press, New York, New York, USA, 146.

13. Enrico Rukzio, Paul Holleis, and Hans Gellersen. 2012. Personal Projectors for Pervasive Computing. IEEE Pervasive Computing 11, 2 (April 2012), 30-37. DOI=http://dx.doi.org/10.1109/MPRV.2011.17

14. Schultz, Michelle. 2013. A case study on the appropriateness of using quick response (QR) codes in libraries and museums. Library \& Information Science Research 35, no. 3 (2013): 207-215. http://dx.doi.org/10.1016/j.lisr.2013.03.002

15. Alan J. Wecker, Joel Lanir, Tsvi Kuflik, and Oliviero Stock. 2011. Pathlight: supporting navigation of small groups in the museum context. In Proceedings of the 13th International Conference on Human Computer Interaction with Mobile Devices and Services (MobileHCI '11). ACM, New York, NY, USA, 569574. DOI=http://dx.doi.org/10.1145/2037373.2037462

16. Max L. Wilson, Dan Craggs, Simon Robinson, Matt Jones, and Kristian Brimble. 2012. Pico-ing into the future of mobile projection and contexts. Personal and Ubiquitous Computing 16, 1 (2012), 39-52. 